\title{
QUASI-LOCAL ENERGY IN PRESENCE OF GRAVITATIONAL RADIATION
}

\author{
PO-NING CHEN, MU-TAO WANG, AND SHING-TUNG YAU
}

\begin{abstract}
We discuss our recent work 4 in which gravitational radiation was studied by evaluating the Wang-Yau quasi-local mass of surfaces of fixed size at the infinity of both axial and polar perturbations of the Schwarzschild spacetime, à la Chandrasekhar [1].
\end{abstract}

We compute the Wang-Yau quasi-local mass [7, 8] of "spheres of unit size" at null infinity to capture the information of gravitational radiation. The set-up, following Chandrasekhar [1], is a gravitational perturbation of the Schwarzschild solution, which is governed by the Regge-Wheeler equation (see below). We take a sphere of a fixed areal radius and push it all the way to null infinity. The limit of the geometric data is that of a standard configuration and thus the optimal embedding equation [7, 8, 2] can be solved.

Let us first consider the axial perturbations. The metric perturbation is of the form:

$$
-\left(1-\frac{2 m}{r}\right) d t^{2}+\frac{1}{1-\frac{2 m}{r}} d r^{2}+r^{2} d \theta^{2}+r^{2} \sin ^{2} \theta\left(d \phi-q_{2} d r-q_{3} d \theta\right)^{2} .
$$

The linearized vacuum Einstein equation is solved by a separation of variable Ansatz in which $q_{2}$ and $q_{3}$ are explicitly given by the Teukolsky function and the Legendre function.

In particular,

$$
q_{3}=\sin (\sigma t) \frac{C_{\mu}(\theta)}{\sin \theta} \frac{\left(r^{2}-2 m r\right)}{\sigma^{2} r^{4}} \frac{d}{d r}\left(r Z^{(-)}\right)
$$

for a solution of frequency $\sigma$ and a separation of variable constant $\mu$. Here $C_{\mu}(\theta)$ is related to the $\mu$-th Legendre function $P_{\mu}$ by

$$
C_{\mu}(\theta)=\sin \theta \frac{d}{d \theta}\left(\frac{1}{\sin \theta} \frac{d P_{\mu}(\cos \theta)}{d \theta}\right) .
$$

Date: October 20, 2018.

P.-N. Chen is supported by NSF grant DMS-1308164, M.-T. Wang is supported by NSF grant DMS-1405152, and S.-T. Yau is supported by NSF grants PHY-0714648 and DMS-1308244. This work was partially supported by a grant from the Simons Foundation (\#305519 to Mu-Tao Wang). Part of this work was carried out when P.-N. Chen and M.T. Wang were visiting the Department of Mathematics and the Center of Mathematical Sciences and Applications at Harvard University. 
After the change of variable

$$
r_{*}=r+2 m \ln \left(\frac{r}{2 m}-1\right),
$$

$Z^{(-)}$satisfies the Regge-Wheeler equation:

$$
\left(\frac{d^{2}}{d r_{*}^{2}}+\sigma^{2}\right) Z^{(-)}=V^{(-)} Z^{(-)},
$$

where

$$
V^{(-)}=\frac{r^{2}-2 m r}{r^{5}}\left[\left(\mu^{2}+2\right) r-6 m\right],
$$

and $\mu$ is a separation of variable constant.

On the Schwarzschild spacetime

$$
-\left(1-\frac{2 m}{r}\right) d t^{2}+\frac{1}{1-\frac{2 m}{r}} d r^{2}+r^{2} d \theta^{2}+r^{2} \sin ^{2} \theta d \phi^{2},
$$

we consider an asymptotically flat Cartesian coordinate system $\left(t, y_{1}, y_{2}, y_{3}\right)$ with $y_{1}=r \sin \theta \sin \phi, y_{2}=r \sin \theta \cos \phi, y_{3}=r \cos \theta$. Given $\left(d_{1}, d_{2}, d_{3}\right) \in \mathbb{R}^{3}$ with $d^{2}=\sum_{i=1}^{3} d_{i}^{2}$, consider the 2 -surface

$$
\Sigma_{t, d}=\left\{\left(t, y_{1}, y_{2}, y_{3}\right): \sum_{i=1}^{3}\left(y_{i}-d_{i}\right)^{2}=1\right\} .
$$

We compute the quasi-local mass of $\Sigma_{t, d}$ as $d \rightarrow \infty$.

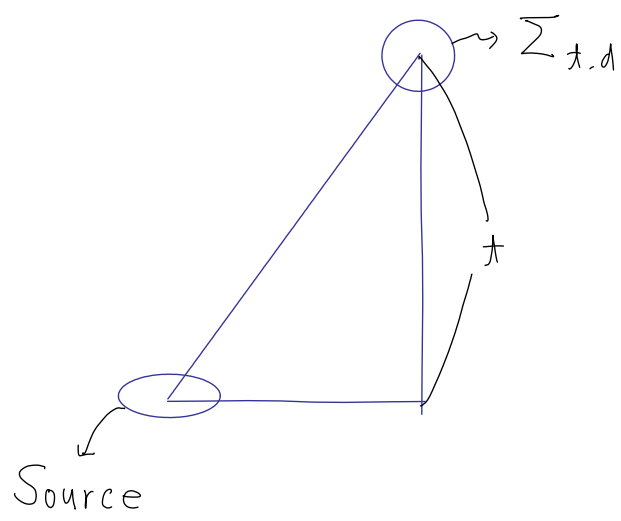

Denote

$$
A(r)=\frac{\left(r^{2}-2 m r\right)}{\sigma^{2} r^{3}} \frac{d}{d r}\left(r Z^{(-)}\right) .
$$


The linearized optimal embedding equation of $\Sigma_{t, d}$ is reduced to two linear elliptic equations on the unit 2-sphere $S^{2}$ :

$$
\begin{aligned}
\Delta(\Delta+2) \tau & =\left[-A^{\prime \prime}\left(1-Z_{1}^{2}\right)+6 A^{\prime} Z_{1}+12 A\right] Z_{2} Z_{3} \\
(\Delta+2) N & =\left(A^{\prime \prime}-2 A^{\prime} Z_{1}+4 A\right) Z_{2} Z_{3},
\end{aligned}
$$

where $\tau$ and $N$ are the respective time and radial components of the solution, and $Z_{1}, Z_{2}, Z_{3}$ are the three standard first eigenfunctions of $S^{2} . A^{\prime}$ and $A^{\prime \prime}$ are derivatives with respect to $r$, and $r^{2}$ is substituted by $r^{2}=d^{2}+2 Z_{1}+1$ in the above equations.

The quasi-local mass of $\Sigma_{t, d}$ with respect to the optimal isometric embedding is then

$$
E\left(\Sigma_{t, d}\right)=C^{2}\left\{\sin ^{2}(\sigma t) E_{1}+\sigma^{2} \cos ^{2}(\sigma t) E_{2}\right\}+O\left(\frac{1}{d^{3}}\right),
$$

where $E_{1}$ and $E_{2}$ are two integrals on the standard unit 2-sphere, that depend on the solution $\tau$ and $N$ of the optimal isometric embedding equation. Explicitly,

$$
\begin{aligned}
& E_{1}=\int_{S^{2}}(1 / 2)\left[A^{2} Z_{2}^{2}\left(7 Z_{3}^{2}+1\right)+2 A A^{\prime} Z_{1} Z_{3}^{2}\left(3 Z_{2}^{2}-1\right)-N(\Delta+2) N\right] \\
& E_{2}=\int_{S^{2}}\left[A^{2} Z_{2}^{2} Z_{3}^{2}-\tau \Delta(\Delta+2) \tau\right]
\end{aligned}
$$

In particular,

$$
\partial_{t} E\left(\Sigma_{t, d}\right)=\frac{\sigma \sin (2 \sigma t) C^{2}(\theta)}{d^{2}}\left\{E_{1}-\sigma^{2} E_{2}\right\}+O\left(\frac{1}{d^{3}}\right)
$$

Let us compare the quasi-local mass on the small spheres $\Sigma_{t, d}$ along a certain direction to the quasi-local mass of the large coordinate spheres $S_{t, r}$.

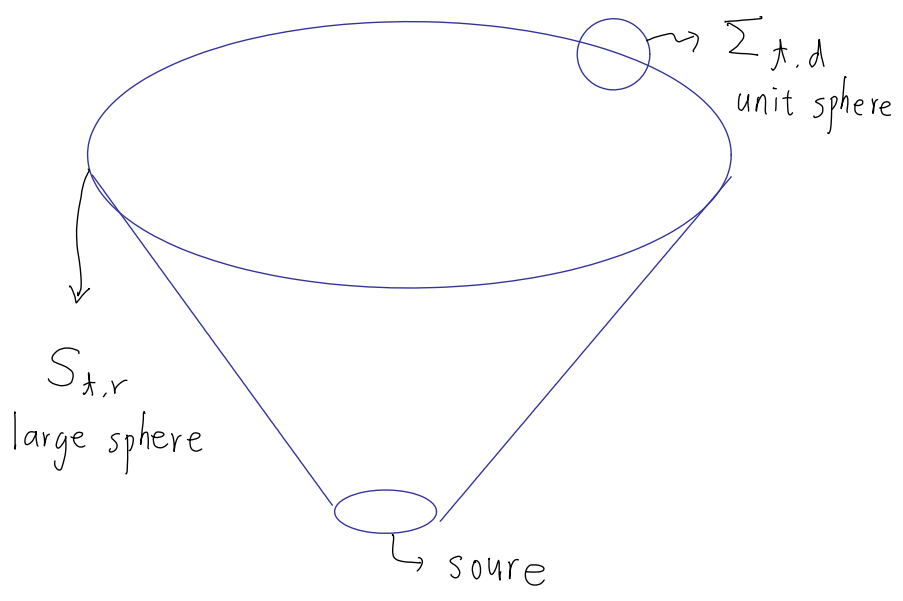


Naively, one may expect to recover $\partial_{t} E\left(S_{t, r}\right)$ by integrating the energy radiated away at all directions $\partial_{t} E\left(\Sigma_{t, d}\right)$. However, our calculation indicates that there are nonlinear correction terms from the quasi-local energy that should be taken into account.

We can also consider the polar perturbation of the Schwarzschild spacetime in which the metric coefficients $g_{t t}, g_{r r}, g_{\theta \theta}$, and $g_{\phi \phi}$ are perturbed in

$$
-\left(1-\frac{2 m}{r}\right) d t^{2}+\frac{1}{1-\frac{2 m}{r}} d r^{2}+r^{2} d \theta^{2}+r^{2} \sin ^{2} \theta d \phi^{2} .
$$

The gravitational perturbation is governed by the Zerilli equation

$$
\left(\frac{d^{2}}{d r_{*}^{2}}+\sigma^{2}\right) Z^{(+)}=V^{(+)} Z^{(+)},
$$

where

$$
V^{(+)}=\frac{2\left(r^{2}-2 m r\right)}{r^{5}(n r+3 m)^{2}}\left[n^{2}(n+1) r^{3}+3 m n^{2} r^{2}+9 m^{2} n r+9 m^{3}\right],
$$

and $n$ is the separation of variable constant. Again, we compute the quasilocal mass of spheres of unit-size at null infinity. The calculation is similar to the axial perturbation case but the result is different as the leading term is of the order $\frac{1}{d}$ (as opposed to $\frac{1}{d^{2}}$ for axial-perturbation) with nonzero coefficients. If such a linear perturbation can be realized as an actual perturbation of the Schwarzschild spacetime, the result would contradict the positivity of the quasi-local mass [6, 7, 8]. From this, we deduce the following conclusion: There does not exist any gravitational perturbation of the Schwarzschild spacetime that is of purely polar type in the sense of Chandrasekhar [1].

For an actual gravitational perturbation of the Schwarzschild solution, the vanishing of the $\frac{1}{d}$ gives a limiting integrand that integrates to zero on the limiting 2 -sphere at null infinity. In fact, the quasi-local mass density $\rho$ (see [3, equation 2.2]) of $\Sigma_{t, d}$ can be computed at the pointwise level. Up to an $O\left(\frac{1}{d^{3}}\right)$ term

$$
\begin{aligned}
\rho & =\left(K-\frac{1}{4}|H|^{2}\right) \\
& -\frac{(|H|-2)^{2}}{4}+\frac{1}{d^{2}}\left\{\frac{1}{2}\left|\nabla^{2} N\right|^{2}+((\Delta+2) N)^{2}-\frac{1}{4}(\Delta N)^{2}\right. \\
& \left.-\frac{1}{4}(\Delta \tau)^{2}+\frac{1}{2}\left[\nabla^{a} \nabla^{b}\left(\tau_{a} \tau_{b}\right)-|\nabla \tau|^{2}-\Delta|\nabla \tau|^{2}\right]\right\},
\end{aligned}
$$

where $K$ is the Gauss curvature of $\Sigma_{t, d}$. The first line, which integrates to zero, is of the order of $\frac{1}{d}$ and is exactly the mass aspect function of the Hawking mass [5]. The $\frac{1}{d^{2}}$ term of the quasi local mass $\int_{\Sigma_{d}} \rho d \mu_{\Sigma_{t, d}}$ has contributions from the second and third lines (of the order of $\frac{1}{d^{2}}$ ), the $\frac{1}{d^{2}}$ term of the first line, and the $\frac{1}{d}$ term of the area element $d \mu_{\Sigma_{t, d}}$. The 
above integral formula is obtained after performing integrations by parts and applying the optimal embedding equation several times.

To each closed loop on the limiting 2 -sphere at null infinity, we can thus associate a non-vanishing arc integral that is of the order of $\frac{1}{d}$, where $d$ is the distance from the source. We expect the freedom in varying the shape of the loop can increase the detectability of gravitational waves.

\section{REFERENCES}

[1] S. Chandrasekhar, The mathematical theory of black holes, reprint of the 1992 edition, Oxford Classic Texts in the Physical Sciences, Oxford Univ. Press, New York.

[2] P.-N. Chen, M.-T. Wang, and S.-T. Yau, Evaluating quasi-local energy and solving optimal embedding equation at null infinity, Comm. Math. Phys. 308 (2011), no.3, 845-863.

[3] P.-N. Chen, M.-T. Wang, and S.-T. Yau, Conserved quantities in general relativity: from the quasi-local level to spatial infinity, Comm. Math. Phys. 338 (2015), no.1, $31-80$.

[4] P.-N. Chen, M.-T. Wang, and S.-T. Yau, Quasi-local mass in the gravitational perturbations of black holes, to appear.

[5] S. W. Hawking, Gravitational radiation in an expanding universe, J. Math. Phys. 9, 598 (1968).

[6] C.-C. M. Liu and S.-T. Yau, Positivity of quasi-local mass II, J. Amer. Math. Soc. 19 (2006), no. 1, 181-204.

[7] M.-T. Wang, and S.-T. Yau, Quasi-local mass in general relativity, Phys. Rev. Lett. 102 (2009), no. 2, no. 021101.

[8] M.-T. Wang, and S.-T. Yau, Isometric embeddings into the Minkowski space and new quasi-local mass, Comm. Math. Phys. 288 (2009), no. 3, 919-942.

Department of Mathematics, Columbia University, New York, NY 10027, USA

E-mail address: mtwang@math.columbia.edu 